\title{
Evolution of speckle during spinodal decomposition
}

\author{
Gregory Brown, ${ }^{1,2}$ Per Arne Rikvold, ${ }^{1}$ Mark Sutton, ${ }^{2}$ and Martin Grant ${ }^{2}$ \\ ${ }^{1}$ Supercomputer Computations Research Institute, Center for Materials Research and Technology, and Department of Physics, \\ Florida State University, Tallahassee, Florida 32306-4130 \\ ${ }^{2}$ Centre for the Physics of Materials, McGill University, 3600 rue University, Montréal, Québec, Canada H3A 2T8
}

(Received 21 May 1999)

\begin{abstract}
Time-dependent properties of the speckled intensity patterns created by scattering coherent radiation from materials undergoing spinodal decomposition are investigated by numerical integration of the Cahn-HilliardCook equation. For binary systems which obey a local conservation law, the characteristic domain size is known to grow in time $\tau$ as $R=[B \tau]^{n}$ with $n=1 / 3$, where $B$ is a constant. The intensities of individual speckles are found to be nonstationary, persistent time series. The two-time intensity covariance at wave vector $\mathbf{k}$ can be collapsed onto a scaling function $\operatorname{Cov}(\delta t, \bar{t})$, where $\delta t=k^{1 / n} B\left|\tau_{2}-\tau_{1}\right|$ and $\bar{t}=k^{1 / n} B\left(\tau_{1}+\tau_{2}\right) / 2$. Both analytically and numerically, the covariance is found to depend on $\delta t$ only through $\delta t / \bar{t}$ in the small- $\bar{t}$ limit and $\delta t / \bar{t}^{1-n}$ in the large- $\bar{t}$ limit, consistent with a simple theory of moving interfaces that applies to any universality class described by a scalar order parameter. The speckle-intensity covariance is numerically demonstrated to be equal to the square of the two-time structure factor of the scattering material, for which an analytic scaling function is obtained for large $\bar{t}$. In addition, the two-time, two-point order-parameter correlation function is found to scale as $C\left(r /\left(B^{n} \sqrt{\tau_{1}^{2 n}+\tau_{2}^{2 n}}\right), \tau_{1} / \tau_{2}\right)$, even for quite large distances $r$. The asymptotic power-law exponent for the autocorrelation function is found to be $\lambda \approx 4.47$, violating an upper bound conjectured by Fisher and Huse. [S1063-651X(99)00311-6]
\end{abstract}

PACS number(s): 64.60.Cn, 64.75.+g, 61.10.Dp, 05.70.Ln

\section{INTRODUCTION}

One of the most common ways to obtain information about the spatial structure of a material involves the scattering of radiation, such as electrons, neutrons, or photons. The scattering is caused by inhomogeneities in the scattering cross section of the material. Depending on the nature of the incident radiation, this scattering cross section is given by the local value of some property of the material, such as its density or magnetic polarization. In the first Born approximation, the amplitude of the scattered wave is given by the Fourier transform of that property, and the wave vector $\mathbf{k}$ is proportional to the momentum transfer as a coherent wave of the probe radiation is scattered. For conventional radiation sources, the wave is only coherent over a small volume and different parts of the sample scatter independently of each other. As a result, only the ensemble average of the scattering intensity can be observed.

On the other hand, when the coherence volume of the wave is sufficiently large to encompass the whole scattering volume, the unaveraged Fourier transform of the specific geometric structure of the material is observed. If the inhomogeneous scattering property of a particular sample at position $\mathbf{r}$ and time $\tau$ is represented as the scalar field $\psi(\mathbf{r}, \tau)$, and its Fourier transform at wave vector $\mathbf{k}$ as $\hat{\psi}(\mathbf{k}, \tau)$, then the observed scattering intensity is

$$
I(\mathbf{k}, \tau)=|\hat{\psi}(\mathbf{k}, \tau)|^{2}
$$

where proportionality constants have been ignored. The domain pattern of the order parameter $\psi(\mathbf{r}, \tau)$ from one of our simulations of spinodal decomposition in a two-phase material is shown in Fig. 1, with the shading differences corre- sponding to differences in the local scattering cross sections. Figure 2 shows the central section of the scattering intensity corresponding to Fig. 1, with darker shades indicating higher intensities. The speckled appearance of the scattering intensity is a characteristic feature in the scattering of coherent radiation.

Since the speckle pattern depends on the specific configuration of the material, it changes as the domain structure evolves over time. The result is that the individual speckles fluctuate around their time-dependent averages. The intensity fluctuations are almost uncorrelated in $\mathbf{k}$ space, but the evo-

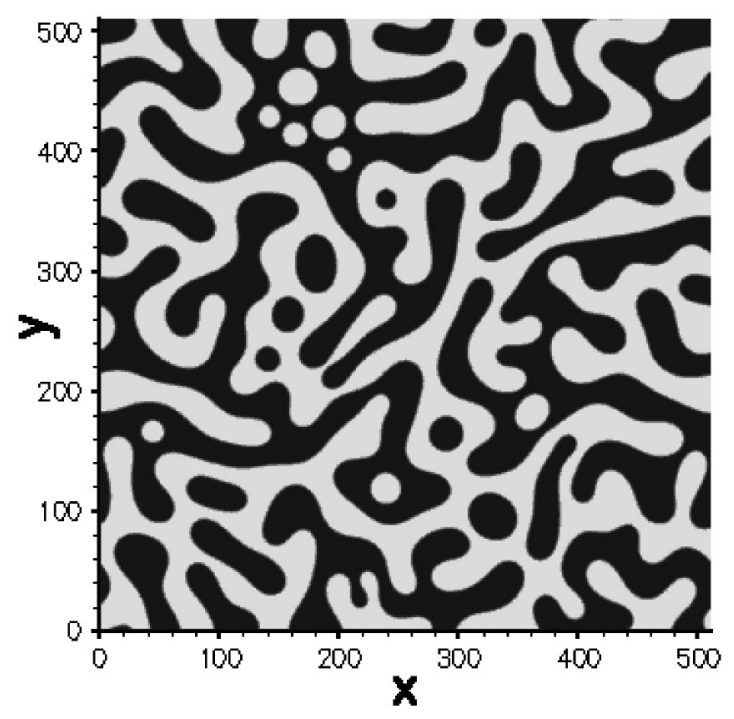

FIG. 1. Snapshot of a system undergoing spinodal decomposition at the maximum simulation time, $\tau=4000$. All simulations reported here were conducted on $512 \times 512$ lattices. 


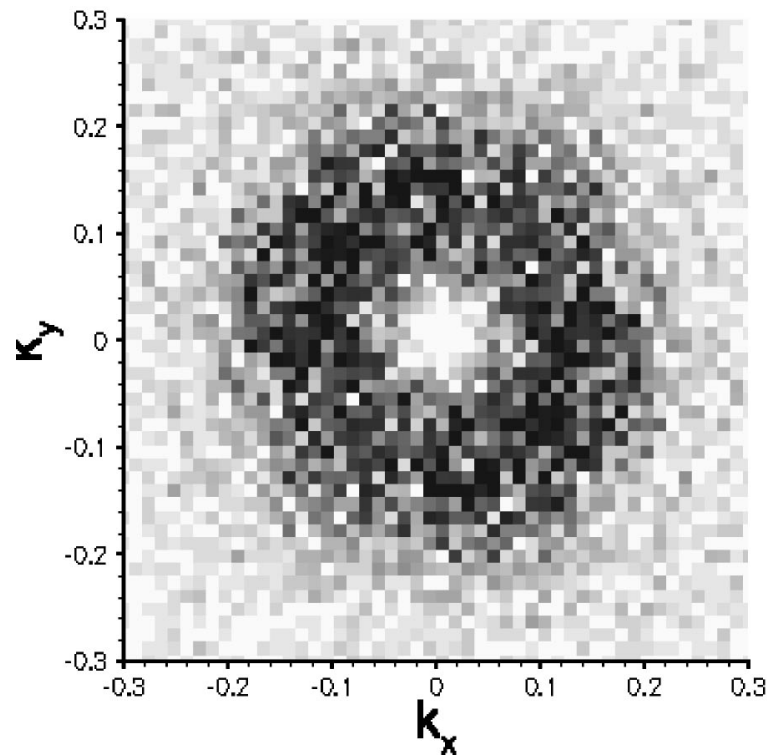

FIG. 2. Central region of the scattering intensity for one realization of the simulation at time $\tau=4000$. The speckling of the scattering pattern is apparent, with darker shades indicating brighter speckles.

lution of the speckle pattern gives rise to nontrivial two-time correlations in the scattering intensity at individual wave vectors.

The fluctuation of a single speckle around the average intensity is shown in Fig. 3. It has been normalized at each time by the corresponding average intensity. Also shown in Fig. 3 are Brownian noise fluctuations, whose two-time covariance is exponential. That noise is constructed to have the same single-time probability density and characteristic time

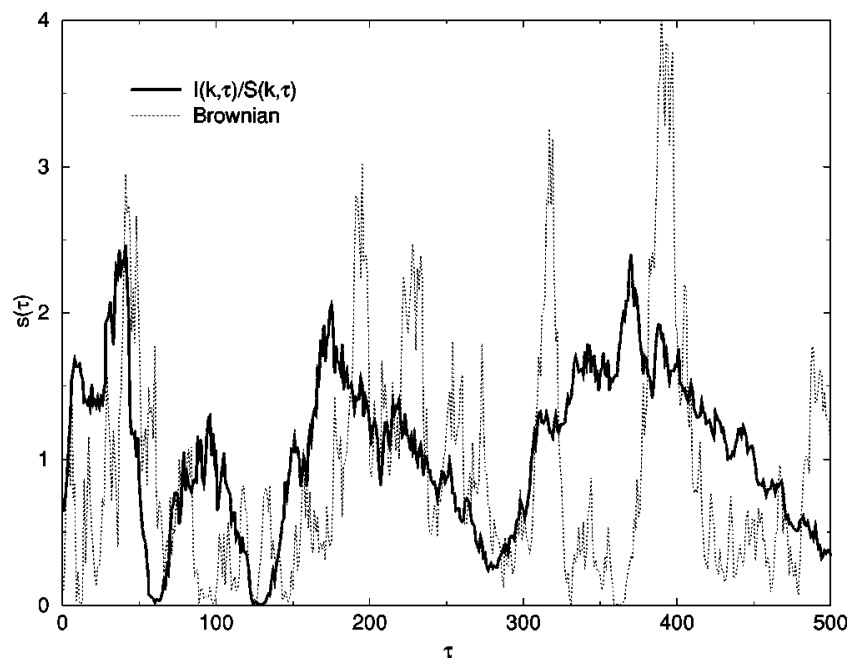

FIG. 3. Time series of the scattering intensity at one wave vector $\mathbf{k}$ for one quench to zero temperature. The intensity has been normalized by the time-dependent structure factor averaged over 100 quenches. The dotted line is a synthetic "Brownian" function constructed to have the same exponential single-time probability density as the scattering intensity and an exponential two-time covariance with a characteristic time corresponding to that of the simulation intensity. The persistence of the scattering-intensity time series is quite apparent when compared to the synthetic Brownian time series. scale as the speckle. There is a clear qualitative difference in time correlations between the two time series. The changes in the Brownian function are relatively large and the fluctuations shorter lived than those of the speckle intensities produced by the phase-ordering simulation. This highlights a property of the speckle intensities called persistence [1]. While the changes in a Brownian time series are independent, the changes in a persistent time series are correlated with each other.

In the past, photon correlation experiments using laser light at wavelengths ranging from the infrared to the ultraviolet have used the two-time correlations of individual speckles to study fluctuations in many materials [2,3]. New high-brilliance synchrotron photon sources have made coherent $\mathrm{x}$-ray experiments feasible. The comparative advantages of $\mathrm{x}$ rays over lasers for many systems include penetration of optically opaque materials and the ability to investigate shorter length scales. Coherent $\mathrm{x}$-ray intensity fluctuation spectroscopy (XIFS) experiments have already been performed on a number of materials [4-7]. Recently, the twotime correlations in speckle intensity were measured by XIFS for a sodium borosilicate glass undergoing spinodal decomposition [8].

Our intent with this paper is to make a theoretical study of how speckle experiments can be exploited to investigate the relaxation of homogeneous materials towards a new, heterogeneous equilibrium after a rapid change in a thermodynamic parameter such as temperature. Previously $[9,10]$ this was done for materials subject to phase ordering, in which case $\psi(\mathbf{r}, t)$ is not constrained by any conservation laws (often called model $A[11])$. Here we analyze scattering from materials subject to a local conservation constraint (model $B$ [11]), such as the process of phase separation in a model binary mixture or alloy. The dynamics of the phase separation that occurs after a high-temperature homogeneous mixture is suddenly brought into the low-temperature regime of two-phase coexistence is a fundamental statisticalmechanical process that has important consequences for multiphase materials. Two examples are metallic alloys [11] and Vycor glass [12].

The scaling properties of correlations in the model system and their relationship to speckle correlations are discussed in Sec. II. Although our focus is on model $B$, many of our results are sufficiently general that they also apply to other universality classes characterized by a scalar order parameter, such as model $A$. Details of the numerical simulations are given in Sec. III. In Sec. IV the numerical results are presented and compared with the theoretical scaling relations from Sec. II. Our conclusions are summarized in Sec. V.

\section{SCALING THEORY}

When the volume fractions of the two components are equal in a binary mixture, the homogeneous hightemperature phase is unstable to long-wavelength fluctuations after the quench. As a consequence, an interpenetrating pattern of domains rich in one or the other of the two components develops immediately after the quench. (See Fig. 1.) This intertwined structure distinguishes spinodal decomposition from many other phase-separation processes. At later times, the domains grow as the system evolves towards an 
equilibrium state with the smallest possible total interfacial area compatible with local conservation of the volume fractions of the constituent phases. The patterns at different times are statistically similar, except for the characteristic length scale of the domains. The description of the late-time regime of phase separation is greatly simplified by this dynamic scaling behavior.

\section{A. Scaling of one-time functions}

Usually, the average size and shape of the growing domains can be measured using the structure factor, which is proportional to the average intensity measured in scattering experiments. For isotropic materials the structure factor depends only on the magnitude of the wave vector,

$$
S(k, \tau)=\langle I(\mathbf{k}, \tau)\rangle,
$$

where the angular brackets represent the ensemble average. For locally conserved dynamics, the structure factor vanishes at both high and low wave vectors. The intervening maximum occurs at $k_{\max }(\tau)$, which is proportional to the inverse of the characteristic domain size. Since the domains grow, the ring of brightest scattering contracts as the system evolves toward equilibrium.

In the scaling regime, the growth of this characteristic length obeys a power law, $R(\tau)=[B \tau]^{n}$, where $B$ is a constant. Such power-law growth is found in many different phase-ordering processes, and the exponent is usually independent of the specific properties of the material. Instead it depends on general features of the phase ordering, such as the presence or absence of local conservation. This dynamical universality picture has been found to be widely applicable, and a group of diverse processes that show common scaling behavior are termed a universality class [11]. For situations described by a scalar order parameter, $n=1 / 2$ when the order parameter is not conserved and $n=1 / 3$ when it is locally conserved.

Dynamical scaling and dynamical universality can be combined to give a single, time-independent description of the statistical structure of phase-separating materials. Since $R(\tau)=[B \tau]^{n}$, a dimensionless scaled wave vector $q$ $=k R(\tau)=k[B \tau]^{n}$ can be defined and the structure factor can be collapsed onto a master curve,

$$
F_{1}^{\prime}(q)=[B \tau]^{-n d} S(k, \tau)
$$

where $d$ is the spatial dimension of the system. Equivalently, a scaled time $t=q^{1 / n}=[k R(\tau)]^{1 / n}=k^{1 / n} B \tau$ gives the master curve

$$
F_{1}(t)=k^{d} S(k, \tau)
$$

where $F_{1}(t)=q^{d} F_{1}^{\prime}\left(t^{n}\right)$. Incoherent scattering experiments are frequently used to measure the average scattering associated with the structure factor.

\section{B. Scaling of two-time functions}

For individual speckles, time-dependent fluctuations around the average intensity are characterized by the twotime intensity covariance function,

$$
\begin{aligned}
\operatorname{Cov}_{\mathbf{k}}\left(\mathbf{k}, \tau_{1}, \tau_{2}\right)= & \left\langle I\left(\mathbf{k}, \tau_{1}\right) I\left(\mathbf{k}, \tau_{2}\right)\right\rangle-\left\langle I\left(\mathbf{k}, \tau_{1}\right)\right\rangle\left\langle I\left(\mathbf{k}, \tau_{2}\right)\right\rangle \\
= & \left\langle\hat{\psi}\left(\mathbf{k}, \tau_{1}\right) \hat{\psi}^{*}\left(\mathbf{k}, \tau_{1}\right) \hat{\psi}\left(\mathbf{k}, \tau_{2}\right) \hat{\psi}^{*}\left(\mathbf{k}, \tau_{2}\right)\right\rangle \\
& -\left\langle\hat{\psi}\left(\mathbf{k}, \tau_{1}\right) \hat{\psi}^{*}\left(\mathbf{k}, \tau_{1}\right)\right\rangle\left\langle\hat{\psi}\left(\mathbf{k}, \tau_{2}\right) \hat{\psi}^{*}\left(\mathbf{k}, \tau_{2}\right)\right\rangle .
\end{aligned}
$$

Following the treatment in Ref. [10], we anticipate that the joint probability density of the complex variables $\hat{\psi}\left(\mathbf{k}, \tau_{1}\right)$ and $\hat{\psi}\left(\mathbf{k}, \tau_{2}\right)$ is Gaussian. The Gaussian nature of the joint probability density, and thus of the marginal probability density for each $\hat{\psi}(\mathbf{k}, \tau)$, is essentially a consequence of the central-limit theorem. It may or may not hold, regardless of the probability density of the order parameter in real space $\psi(\mathbf{r}, \tau)$. Specifically, it does not depend on whether $\psi(\mathbf{r}, \tau)$ can be derived from an underlying Gaussian auxiliary field, an assumption which is customarily referred to as "the Gaussian approximation." In this paper we are only concerned with the former approximation.

It is possible to construct a set a variables whose joint probability density is not Gaussian even though the marginal probability densities are [13], so the additional constraint that the joint probability density be Gaussian is an important one. The essential aspect of this assumption is that the fourth moment which occurs in Eq. (5) reduces to products of second moments according to Wick's theorem. For this reason we refer to the approximation as the "Gaussian decoupling approximation.', For $\mathbf{k} \neq \mathbf{0}$ the covariance becomes equal to the square of the two-time structure factor [10],

$$
\operatorname{Cov}_{\mathbf{k}}\left(\mathbf{k}, \tau_{1}, \tau_{2}\right)=S^{2}\left(\mathbf{k}, \tau_{1}, \tau_{2}\right)
$$

The two-time structure factor is defined as

$$
S\left(\mathbf{k}, \tau_{1}, \tau_{2}\right) \equiv\left\langle\hat{\psi}\left(\mathbf{k}, \tau_{1}\right) \hat{\psi}^{*}\left(\mathbf{k}, \tau_{2}\right)\right\rangle,
$$

but it is also proportional to the Fourier transform of the two-point, two-time order-parameter correlation function

$$
S\left(\mathbf{k}, \tau_{1}, \tau_{2}\right)=\int d \mathbf{r} e^{i \mathbf{k} \cdot \mathbf{r}} C\left(\mathbf{r}, \tau_{1}, \tau_{2}\right)
$$

where the correlation function is defined by

$$
C\left(\mathbf{r}, \tau_{1}, \tau_{2}\right)=\left\langle\psi\left(\mathbf{0}, \tau_{1}\right) \psi\left(\mathbf{r}, \tau_{2}\right)\right\rangle
$$

Similar to the case for the one-time structure factor, dynamical scaling also applies to the two-time structure factor. For isotropic media, the latter can be related to a master function of the two scaled times,

$$
F_{2}\left(t_{1}, t_{2}\right)=k^{d} S\left(k, \tau_{1}, \tau_{2}\right) .
$$

Since it involves the Fourier transform of the material at two different times, the two-time structure factor cannot be measured directly in a scattering experiment. However, its relationship to the two-time intensity covariance indicates that it can be inferred from the fluctuations of speckles around their average intensities. In analogy with the scaling of the twotime structure factor, a scaled speckle covariance

$$
\operatorname{Cov}\left(t_{1}, t_{2}\right)=k^{2 d} \operatorname{Cov}_{\mathrm{k}}\left(k, \tau_{1}, \tau_{2}\right)
$$




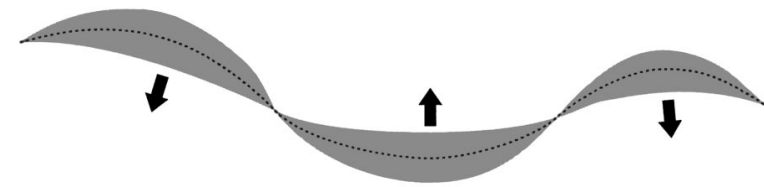

FIG. 4. Schematic illustration of the moving-interface model. The edges of the shaded region represent the interface at times $\tau_{1}$ and $\tau_{2}$, and the arrows indicate the directions of motion. The dashed curve is the mean interface. The shaded region represents the volume fraction $\phi_{\Delta}$, which changes phase between $\tau_{1}$ and $\tau_{2}$.

can be defined. Then

$$
\operatorname{Cov}\left(t_{1}, t_{2}\right)=F_{2}^{2}\left(t_{1}, t_{2}\right)
$$

provided the relationship between the speckle covariance and the two-time structure factor, Eq. (6), is valid.

The asymptotic properties of the two-time structure factor can be found using quite general arguments that should apply for many universality classes. They are most concretely expressed by focusing on the geometry of the moving interfaces, a schematic view of which is provided in Fig. 4. The top and bottom of the figure remain inside the domains of the two different phases, respectively, while the shaded region changes phase between $\tau_{1}$ and $\tau_{2}$. The edges of the shaded region represent the interface between the domains at $\tau_{1}$ and $\tau_{2}$; the interface at $\tau_{2}$ is flatter. Roughly halfway between these interfaces, the dashed line represents the "mean interface," which defines a mean domain size, $[B \bar{\tau}]^{n}$, where $\bar{\tau}$ $=\left(\tau_{2}+\tau_{1}\right) / 2$. Lengths are scaled by this mean domain size. Using this specific choice of scaling length, along with the ansatz that the times appear as the ratio $\tau_{2} / \tau_{1}$, the relationship between the two-time order-parameter correlation function and its master curve can be written

$$
C\left(\mathbf{r}, \tau_{1}, \tau_{2}\right)=H\left(\mathbf{r} /[B \bar{\tau}]^{n}, \delta \tau / \bar{\tau}\right),
$$

where $\delta \tau=\left|\tau_{2}-\tau_{1}\right|$.

The master curve for the two-time structure factor can be found using Eq. (8). When the correlation function is isotropic, this can be reduced to a radial integral that depends on the scaled-time variables $\delta t=\left|t_{2}-t_{1}\right|$ and $\bar{t}=\left(t_{1}+t_{2}\right) / 2$;

$$
\begin{aligned}
F_{2}(\delta t, \bar{t})= & (2 \pi)^{d / 2} \bar{t}^{n(d / 2+1)} \\
& \times \int_{0}^{\infty} d u u^{d / 2} J_{d / 2-1}\left(u \bar{t}^{n}\right) H(u, \delta t / \bar{t}),
\end{aligned}
$$

where $J_{\nu}$ is a Bessel function of the first kind of order $\nu$.

The small $-\bar{t}$ and large- $\bar{t}$ behaviors of $F_{2}$ can both be found using elements of the picture described here.

\section{Two-time scaling for small $\bar{t}$}

The small- $\bar{t}$ behavior of Eq. (14) is isolated by Taylor expanding the Bessel function,

$$
F_{2}(\delta t, \bar{t})=2 \pi^{d / 2} \bar{t} n d \sum_{j=0}^{\infty}(-1)^{j} \bar{t}^{2 n j} h_{2 j}(\delta t / \bar{t}),
$$

where $h_{2 j}(\delta t / \bar{t})$ is defined through the integration over the scaled distance $u$,

$$
h_{2 j}(\delta t / \bar{t})=\frac{\int_{0}^{\infty} d u u^{2 j+d-1} H(u, \delta t / \bar{t})}{4^{j} j ! \Gamma(j+d / 2)} .
$$

At small wave vector the structure factor can be shown to obey a power law $S \propto k^{\beta(n)}$, or equivalently $F_{2} \propto \bar{t}^{n[\beta(n)+d]}$. The value is $\beta(1 / 2)=0$ for model $A$ [14] and is believed to be $\beta(1 / 3)=4$ for model $B$ [15]. For model $B$, this indicates that $h_{0}(0)=h_{2}(0)=0$ and, consequently, that the leading term is the one containing $h_{4}(\delta t / \bar{t})$. The Taylor expansion is well approximated by its leading term when that term is much larger than the one containing $h_{6}$. This condition is equivalent to

$$
\bar{t}^{2 n} \ll 12\left(\frac{d}{2}+2\right) \frac{\int_{0}^{\infty} d u u^{d+3} H(u, \delta t / \bar{t})}{\int_{0}^{\infty} d u u^{d+5} H(u, \delta t / \bar{t})},
$$

where the radio of the integrals is a function of $\delta t / \bar{t}$. For $\bar{t}$ small enough, then, the scaling of the two-time structure factor depends on $\delta t$ only through $\delta t / \bar{t}$. The analogous result for model $A$ [which follows with $h_{0}(0)>0$ ] was already obtained explicitly in Ref. [10] for the Yeung-Jasnow (YJ) [16] correlation function. This small- $\bar{t}$ behavior agrees with our numerical results for both model $A[10]$ and model $B$ (see Sec. IV).

\section{Two-time scaling for large $\bar{t}$}

When Eq. (17) is not satisfied, a large number of terms is required in Eq. (15) to obtain an accurate estimate of $F_{2}(\delta t, \bar{t})$. Since these terms contain products of powers of $\bar{t}$ and $\delta t / \bar{t}, F_{2}(\delta t, \bar{t})$ should not be expected to depend on $\delta t$ only through $\delta t / \bar{t}$ for large $\bar{t}$. In Ref. [10] this was demonstrated explicitly for model $A$ using the YJ [16] analytic approximation for the correlation function. The result presented here is based on simple geometric arguments, which should be valid for all universality classes with a scalar order parameter, including both model $A$ and model $B$.

At large wave vectors the one-time structure factor for a two-phase system with sharp, randomly oriented interfaces is well described by Porod's law [17], $S \propto k^{-(d+1)}$. Here the proportionality constant includes the interface area per unit volume (the specific surface) at time $\tau, A(\tau) \propto 1 / R(\tau)$. In this limit the scattering probes the correlation function at lengths $r \ll R(\tau)$, where the normalized equal-time correlation function decreases linearly with scaled distance $A(\tau) r$ $\propto r / R(\tau)[18-20]$,

$$
\frac{C(\mathbf{r}, \tau, \tau)}{\left\langle\psi^{2}(\tau)\right\rangle}=1-C_{1} \frac{r}{[B \tau]^{n}} .
$$

Here $C_{1}$ is a nonuniversal constant. The mean-square order parameter $\left\langle\psi^{2}(\tau)\right\rangle$ is independent of $\tau$ if the two equilibrium 
values of $\psi$ are equal in magnitude or the volume fractions of the two phases are constant, as they are in model $B$, and we will simply denote it $\left\langle\psi^{2}\right\rangle$.

The behavior for small, but nonzero, time differences can be deduced from the moving-interface model. The normalized two-time autocorrelation function $C\left(\mathbf{0}, \tau_{1}, \tau_{2}\right) /\left\langle\psi^{2}\right\rangle$ is reduced from unity by an amount proportional to $\phi_{\Delta}$, the fraction of the system volume which is occupied by different phases at $\tau_{1}$ and $\tau_{2}$, i.e., the shaded volume in Fig. 4 . In the sharp-interface picture used here, this is an exact result, obtained by simple probability arguments. The proportionality constant is easily calculated but not very useful.

For small time differences in a moving-interface model, one can construct a coordinate system based on the mean interfacial position, represented by the dashed curve in Fig. 4 , and a coordinate locally perpendicular to it, $\Delta$. The specific surface of the mean interface is approximately $\bar{A}$ $\approx\left[A\left(\tau_{1}\right)+A\left(\tau_{2}\right)\right] / 2$, and the average distance between the other interfaces is $\langle|\Delta|\rangle$. In this approximation, $\phi_{\Delta}$ $\approx \bar{A}\langle|\Delta|\rangle$. Using the scaling assumption that $R(\tau)=[B \tau]^{n}$ $\propto 1 / A(\tau)$ is the only length scale characterizing the system at time $\tau$, we obtain

$$
\left.\langle|\Delta|\rangle \propto\left|R\left(\tau_{2}\right)-R\left(\tau_{1}\right)\right| \approx \frac{d R(\tau)}{d \tau}\right|_{\tau=\bar{\tau}} \delta \tau=n B^{n} \frac{\delta \tau}{\bar{\tau}^{1-n}} \equiv n B^{n} \zeta .
$$

The dimensionless proportionality constant between $\langle|\Delta|\rangle$ and $\left|R\left(\tau_{2}\right)-R\left(\tau_{1}\right)\right|$ is expected to depend on the particular dynamic model and the dimensionality $d$. The relation $\left|R\left(\tau_{2}\right)-R\left(\tau_{1}\right)\right| \approx n B^{n} \zeta$ is a Taylor expansion valid for small $\zeta$. These results can be combined to give

$$
\frac{C\left(\mathbf{0}, \tau_{1}, \tau_{2}\right)}{\left\langle\psi^{2}\right\rangle}=1-\frac{n C_{1} C_{2} \zeta}{\bar{\tau}^{n}},
$$

where $C_{2}$ contains the proportionality constants from Eq. (19).

On length scales $\langle|\Delta|\rangle \ll r \ll 1 / \bar{A}$ the interface should appear sharp, so the asymptotic correlation function should be described using the mean domain size, i.e., by Eq. (18) with $\tau^{-n}$ replaced by $\bar{\tau}^{-n}$. The crossover between the small- $r$ and intermediate- $r$ limits can be described in terms of a scaling function $G(x)$ as

$$
\frac{C(\mathbf{r}, \delta \tau, \bar{\tau})}{\left\langle\psi^{2}\right\rangle}=1-\frac{n C_{1} C_{2} \zeta}{\bar{\tau}^{n}} G\left(\frac{r}{n C_{2} B^{n} \zeta}\right),
$$

with the asymptotic behavior

$$
G(x) \sim \begin{cases}1 & \text { for } x \ll 1 \\ x & \text { for } x \gg 1\end{cases}
$$

Thus, $C(\mathbf{r}, \delta \tau, \bar{\tau})$ depends on $r$ only through the dimensionless scaling combination $r /\left[n C_{2} B^{n} \zeta\right]$. As seen from the derivation, this result is general and applies to any movinginterface model.
The two-time structure factor in the large- $k$ limit is obtained from Eq. (21) using the same formula for the $d$-dimensional Fourier transform of an isotropic function as in Eq. (14),

$$
\begin{aligned}
S\left(k, \tau_{1}, \tau_{2}\right)= & -k^{-d}\left\langle\psi^{2}\right\rangle(2 \pi)^{d / 2} \frac{n C_{1} C_{2} \zeta}{\bar{\tau}^{n}} \\
& \times \int_{0}^{\infty} d u u^{d / 2} J_{d / 2-1}(u) G\left(\frac{u}{n C_{2} k B^{n} \zeta}\right),
\end{aligned}
$$

where we have removed from $C(\mathbf{r}, \delta \tau, \bar{\tau})$ the constant term which only contributes to a $\delta$ function at $k=0$. Defining the new dimensionless scaling variable $z=k B^{n} \zeta \equiv \delta t / \bar{t}^{1-n}$, we obtain the asymptotic scaling form for the two-time structure factor in the large- $\bar{t}$ limit:

$$
\begin{aligned}
\Phi_{d}(z) \equiv \frac{\bar{t}^{n} F_{2}(z, \bar{t})}{\left\langle\psi^{2}\right\rangle C_{1}}= & -(2 \pi)^{d / 2} n C_{2} z \\
& \times \int_{0}^{\infty} d u u^{d / 2} J_{d / 2-1}(u) G\left(\frac{u}{n C_{2} z}\right) .
\end{aligned}
$$

This asymptotic scaling function depends on $k, \tau_{1}$, and $\tau_{2}$ only through $z$. If the correlation function vanishes sufficiently smoothly for large $r$, standard expansions of Fourier transforms $[20,21]$ indicate that the asymptotic large- $k$ behavior of the structure factor is determined completely by the real-space behavior of the correlation function for small $r$. For $z=0$ it reduces to the single-time scaling function [20]

$$
\Phi_{d}(0)=\frac{\bar{t}^{n} F_{2}(0, \bar{t})}{C_{1}}=2^{d} \pi^{(d-1) / 2} \Gamma\left(\frac{d+1}{2}\right),
$$

which is consistent with the amplitude of the large- $k$ Porod tail of the structure factor $\left[\Phi_{2}(0)=2 \pi\right.$ and $\left.\Phi_{3}(0)=8 \pi\right]$. As $z$ increases, the integral in Eq. (23) tends towards the Fourier transform of a constant, which vanishes for all nonzero $k$. Thus, $\Phi_{d}(z)$ decreases from its maximum value at $z=0$ towards zero as $z$ increases.

In Ref. [10] the scaling behavior expressed in Eq. (23) was explicitly obtained for model $A$, but a nonrigorous scaling argument suggested it should hold for other dynamical models as well. This was recently confirmed by XIFS experiments in a sodium borosilicate glass undergoing spinodal decomposition [8]. This is a two-phase system with a locally conserved scalar order parameter appropriately described by model $B$.

To predict the form of $\Phi_{d}(z)$ in further detail, it is necessary to know how $G(x)$ interpolates between the limiting behaviors given by Eq. (21b). A detailed calculation based on an extension of an approach used by Tomita to calculate equal-time correlation functions $[19,20]$ indicates that $G(x)$ $-x \propto x^{-1}$ as $x \rightarrow \infty$ and $G(x)-1 \propto x^{2}$ as $x \rightarrow 0$ for movinginterface models [22]. A convenient analytic form with this behavior is $G(x)=\sqrt{1+x^{2}}$. Choosing $\mathrm{v}=n C_{2} z$ and the substitution $u^{2}+\mathrm{v}^{2}=(\mathrm{v} y)^{2}$, the integral $I(\mathrm{~V}) \equiv \Phi_{d}\left(\mathrm{~V} / n C_{2}\right)$ is converted to [23] 


$$
\begin{aligned}
I(\mathrm{v})= & -(2 \pi)^{d / 2} \int_{0}^{\infty} d u u^{d / 2} J_{d / 2-1}(u) \sqrt{u^{2}+\mathrm{v}^{2}} \\
= & -(2 \pi)^{d / 2} \mathrm{v}^{d / 2+2} \int_{1}^{\infty} d y y^{2}\left(\sqrt{y^{2}-1}\right)^{d / 2-1} \\
& \times J_{d / 2-1}\left(\mathrm{v} \sqrt{y^{2}-1}\right) .
\end{aligned}
$$

Asymptotic convergence is guaranteed by the discussion following Eq. (24), and it is possible to use the approach of evaluating a generating function for the desired integral as an Abel limit [23,24],

$$
\begin{aligned}
I(\mathrm{v})= & -(2 \pi)^{d / 2} \mathrm{v}^{d / 2+2} \lim _{\alpha \rightarrow 0} \frac{\partial^{2}}{\partial \alpha^{2}} \\
& \times \int_{1}^{\infty} d y e^{-\alpha y}\left(\sqrt{y^{2}-1}\right)^{d / 2-1} J_{d / 2-1}\left(\mathrm{v} \sqrt{y^{2}-1}\right) .
\end{aligned}
$$

This integral can be found in tables [25], and differentiation followed by taking the limit leads to

$$
\Phi_{d}(z)=\Phi_{d}(0) \frac{\left(n C_{2} z\right)^{(d+1) / 2} K_{(d+1) / 2}\left(n C_{2} z\right)}{2^{(d-1) / 2} \Gamma((d+1) / 2)},
$$

where $K_{\nu}$ is a modified Bessel function of the second kind. For $d=3$ this can be numerically evaluated without difficulty, while for $d=2$ the expression simplifies further to [26]

$$
\Phi_{2}(z)=2 \pi\left(1+n C_{2} z\right) e^{-n C_{2} z} .
$$

The same result was obtained in Ref. [10], using the full YJ [16] correlation function for model $A$ (see the Appendix). The advantage of the present approach is that it demonstrates that $\Phi_{d}(z)$ is manifestly independent of the large- $r$ behavior of the correlation function, requiring only that it converges sufficiently smoothly to ensure the equality of the Abel limit and the integral.

\section{NUMERICAL PROCEDURE}

In phase-separating systems no material is created or destroyed. The order parameter is locally conserved, and evolution occurs by diffusion along chemical-potential gradients. The Cahn-Hilliard-Cook model $[27,28]$ is a convenient description for the dynamics of a conserved scalar order parameter. The thermodynamics of the system are described by the Ginzburg-Landau-Wilson free energy [11],

$$
\begin{aligned}
\mathcal{F} \psi(\mathbf{r}, \tau)]= & \int d \mathbf{r}\left[-\frac{a}{2} \psi^{2}(\mathbf{r}, \tau)+\frac{u}{4} \psi^{4}(\mathbf{r}, \tau)\right. \\
& \left.+\frac{c}{2}|\nabla \psi(\mathbf{r}, \tau)|^{2}\right] .
\end{aligned}
$$

For $a>0$, the first two terms of the integrand create a bistable local potential energy. The last term represents the surface tension between domains in which $\psi$ has opposite sign. The dynamics are implemented using the Langevin equation

$$
\frac{\partial \psi(\mathbf{r}, \tau)}{\partial \tau}=M \nabla^{2} \frac{\delta \mathcal{F}[\psi(\mathbf{r}, \tau)]}{\delta \psi(\mathbf{r}, \tau)}+\eta(\mathbf{r}, \tau) .
$$

The first term on the right-hand side represents deterministic relaxation of the chemical potential; the Laplacian expresses the local conservation constraint. Processes operating at shorter time and length scales are considered thermal noise and are modeled by the random variable $\eta$, whose intensity is given by a fluctuation-dissipation theorem [11]. We neglect $\eta$ because the most important sources of noise here are the initial conditions, which give the random domain morphology at early times.

For the symmetric mixtures considered here, the parameters can be eliminated by appropriately normalizing the time, length, and concentration scales [10,29,30]. Using the same names for the new variables, the resulting dynamical equation is

$$
\frac{\partial \psi(\mathbf{r}, \tau)}{\partial \tau}=-\frac{1}{2} \nabla^{2}\left[\left(1+\nabla^{2}\right) \psi(\mathbf{r}, \tau)-\psi^{3}(\mathbf{r}, \tau)\right]
$$

In particular, this yields $\psi= \pm 1$ for the equilibrium values of the order parameter.

We have simulated this model on a square lattice, with $\Delta r=1$ and $L_{x}=L_{y}=L=512$, using a simple Euler integration scheme with $\Delta \tau=0.05$. The initial condition was implemented by choosing random values uniformly distributed between \pm 0.1 for each point on the lattice. Measurements were made every 50 time units out to a maximum of $\tau=4000$. As can be seen from Fig. 1, the domains are much smaller than the system size, even at this latest time. Finite-size effects are therefore not expected to affect the order-parameter dynamics. Indeed, no deviations from the expected behavior of the characteristic length are observed in the simulations.

The other numerical procedures are identical to those described in Ref. [10]. The Laplacian was implemented using eight-neighbor discretization [31,32], and the magnitude of the wave vector $k(\mathbf{k})$ was defined in a manner consistent with that Laplacian. In addition, the Fourier transform of the hardened order parameter, $\operatorname{sgn}[\psi(\mathbf{r}, \tau)]$, was used to minimize the effect of finite interface width on the scattering intensity.

\section{RESULTS}

The Gaussian decoupling approximation that leads to Eq. (6) has not been directly justified for phase-ordering systems. Gaussian variables are often assumed in the context of the central limit theorem, where it can be argued that many independent random variables with finite variance contribute additively. Since $R(\tau)$ represents the average domain size, at any given time there are on the order of $[L / R(\tau)]^{d}$ independent domains in a system of edge length $L$. This number can be quite large, but since the domains interact as they grow, with material diffusing along chemical-potential gradients, the applicability of the central limit theorem is not obvious. In our previous study of a system with nonconserved order parameter [10], the decoupling was justified a posteriori from the numerical results. Similar justification is presented here for numerical integration of the equation of motion for the conserved order parameter. When discussing the numeri- 


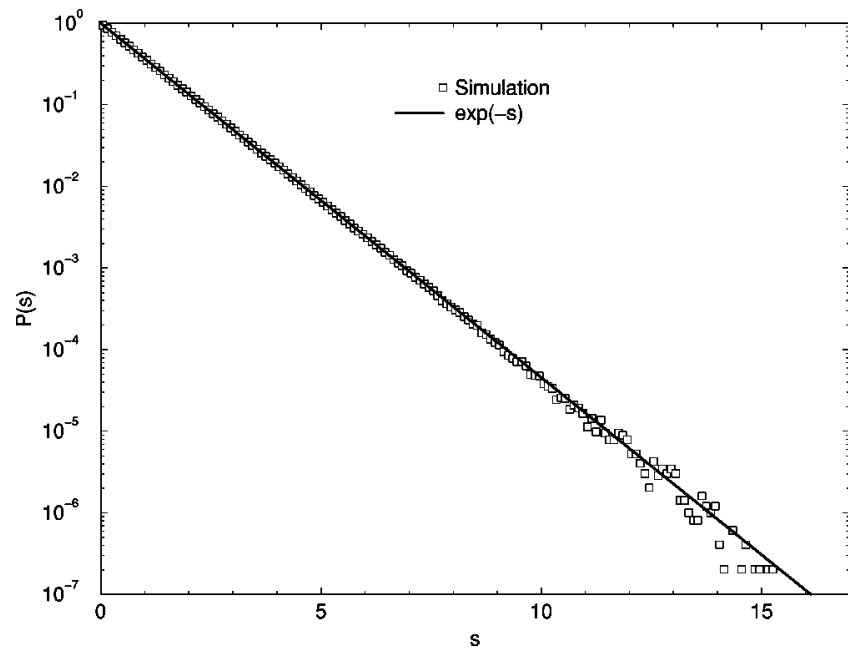

FIG. 5. Probability density of the normalized scattering intensity $s(k, \tau)=I(k, \tau) / S(k, \tau)$, binned for all times and $0.08<k(\mathbf{k})$ $<0.75$. The histogram is exponential for all $s$ observed here, indicating the Gaussian decoupling approximation used to equate the squared two-time structure factor with the speckle covariance is a reasonable approximation at the one-time level.

cal results, we have taken $B=1$ for convenience.

One way to test the validity of the assumption at the single-time level is by looking at the probability density of the speckle intensities. Namely, Eq. (6) with $\tau_{1}=\tau_{2}$ is satisfied if the normalized scattering intensity, $s(\mathbf{k}, \tau)$ $=I(\mathbf{k}, \tau) / S(\mathbf{k}, \tau)$, has an exponential probability density,

$$
P(s)=\exp (-s)
$$

that is independent of $(\mathbf{k}, \tau)$. A single histogram was constructed for the normalized intensity for all times and for $0.08<k(\mathbf{k})<0.75$ [33]. The intensity at a particular $(k, \tau)$ was normalized by $S(k, \tau)$ estimated by circular averaging only for the same quench experiment. The lower $k$ cutoff was chosen so that at least 20 speckles contributed to that circular average, while the upper cutoff was set using results for $\tau_{1}$ $\neq \tau_{2}$ (discussed below). Nearly $10^{8}$ samples contribute to the histogram in Fig. 5, and the agreement with the exponential form is remarkable. Other histograms were constructed to check for $k$ and $\tau$ dependence, but are not shown here. Wave-vector dependence was investigated by sampling from narrow rings of constant $k$ at all times, and time dependence was checked by sampling all $k$ between the cutoffs at single times. The probability density for the normalized intensity does not appear to depend on these variables. A similar check of the Gaussian decoupling assumption was made by Shinozaki and Oono [34] for a cell-dynamical simulation with conserved order parameter.

The equality between the two-time structure factor squared and the two-time intensity covariance implied by the Gaussian decoupling assumption can also be tested directly. These two quantities can be estimated from the simulations using Eq. (7) and Eq. (5), respectively, with circular averaging over $\mathbf{k}$ in addition to the averaging over the quenches. Since the real-space correlation function is an even function of $\mathbf{r}, S\left(\mathbf{k}, \tau_{1}, \tau_{2}\right)$ is real valued. We have verified that the imaginary part is zero to within the accuracy of our results. The equality is tested graphically in Fig. 6 as a function of
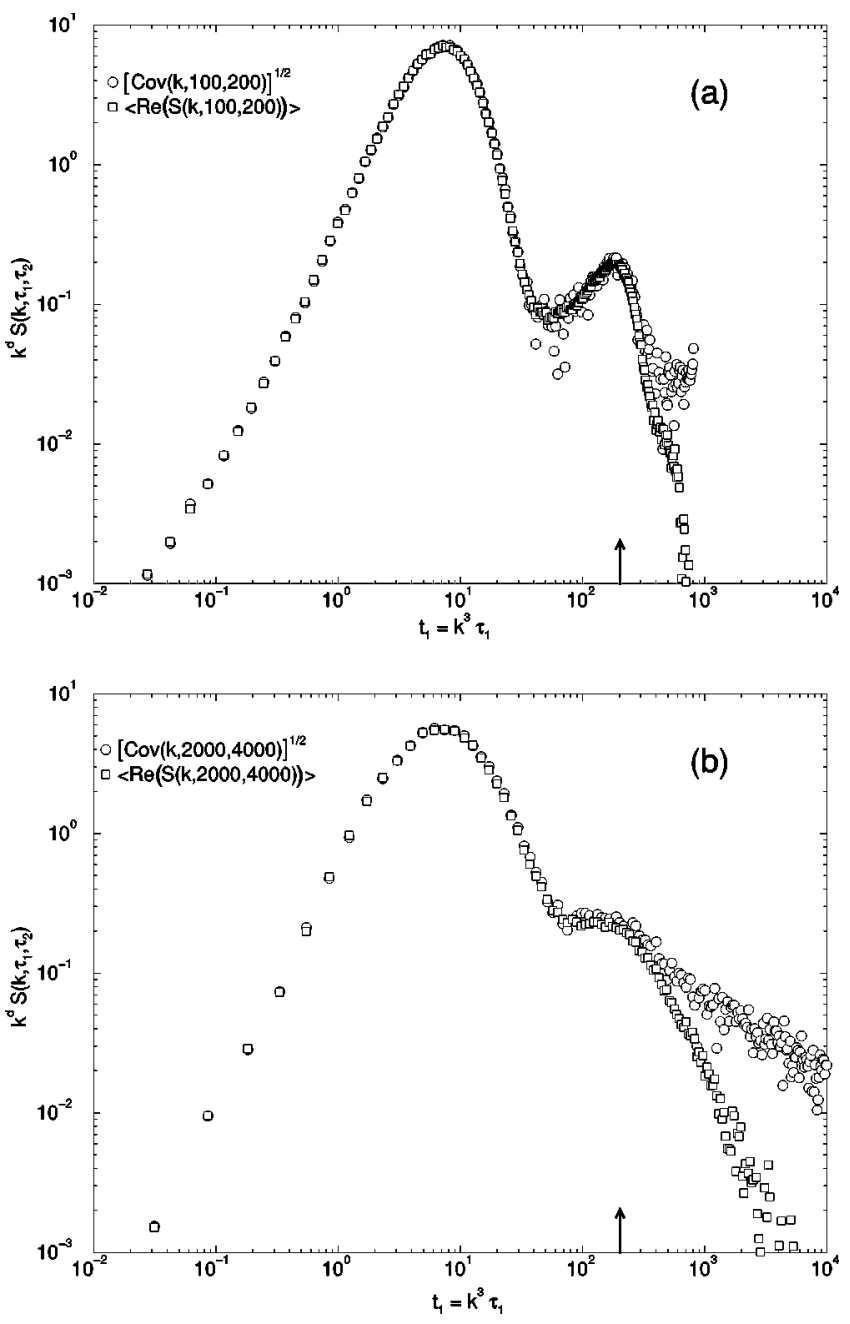

FIG. 6. Comparison of the two-time structure factor $S\left(k, \tau_{1}, \tau_{2}\right)$ (squares) and the square root of the covariance, $\sqrt{\operatorname{Cov}_{\mathbf{k}}\left(k, \tau_{1}, \tau_{2}\right)}$ (circles) for (a) $\tau_{1}=100, \tau_{2}=200$ and (b) $\tau_{1}=2000, \tau_{2}=4000$. These examples serve as a direct test of Eq. (6). The agreement is good for small wave vectors, suggesting that two-time correlation functions for real systems can be measured via speckle experiments. The arrows indicate the scaling variable $z \approx 4.5$ where the Gaussian decoupling for two-time correlations breaks down in these simulations. For $\tau_{2} / \tau_{1}=2$, as in this figure, this corresponds to $k^{3} \tau_{1}$ $\approx 190$.

$t_{1}=k^{3} \tau_{1}$ for (a) $\tau_{1}=100, \tau_{2}=200$ and (b) $\tau_{1}=2000, \tau_{2}$ $=4000$. The intensity covariance and the squared structure factor are equal within our numerical accuracy for sufficiently small $k$. However, for $z \gtrsim 4.5$ (marked by arrows in both parts of Fig. 6) the covariance becomes larger than the squared structure factor, indicating the gradual breakdown of the Gaussian decoupling approximation for large $z$ in these simulations. A systematic investigation of the range of validity of the Gaussian decoupling approximation, including the possible system-size dependence of the number of independent contributions to the Fourier transfom, is left for future study. In this paper all quantitative scaling results are based only on data for $k(\mathbf{k})<0.75$ [33], where the squared twotime structure factor appears to equal the speckle covariance for all times considered.

The scaling function $F_{2}\left(t_{1}, t_{2}\right)$, which describes both the two-time structure factor and the related speckle covariance, 


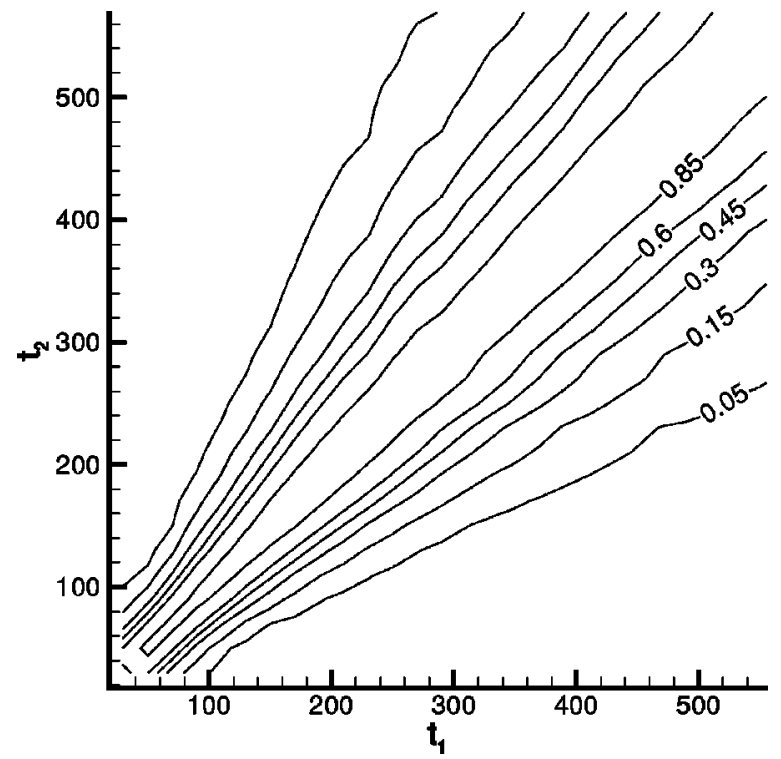

FIG. 7. Contour plot of the scaled speckle two-time correlation function $\operatorname{Corr}\left(t_{1}, t_{2}\right)$. The correlation decreases as one moves away from the line $t_{1}=t_{2}$, where it is unity by construction.

depends on two rescaled times. A normalized analog of the scaled covariance, the correlation function, can be defined as

$$
\operatorname{Corr}\left(t_{1}, t_{2}\right)=\frac{\left\langle I\left(k, \tau_{1}\right) I\left(k, \tau_{2}\right)\right\rangle}{\left\langle I\left(k, \tau_{1}\right)\right\rangle\left\langle I\left(k, \tau_{2}\right)\right\rangle}-1
$$

Since the Gaussian decoupling approximation for scattering has been verified for $\tau_{1}=\tau_{2}$, this quantity is unity by construction for $t_{1}=t_{2}$. Thus the contour plot of $\operatorname{Corr}\left(t_{1}, t_{2}\right)$, Fig. 7, is more illustrative than one for $\operatorname{Cov}\left(t_{1}, t_{2}\right)$. The correlation function decays as one moves away from the $t_{1}$ $=t_{2}$ diagonal. Given the symmetry under exchange of $t_{1}$ and $t_{2}, \bar{t}$ and $\delta t$ are more natural variables for the scaling functions Corr and Cov. In particular, $\bar{t}$ measures distance (in units of scaled time) along the diagonal, while $\delta t$ measures distance perpendicular to the diagonal. In Fig. 7 it is apparent that the speckle intensity stays correlated for larger $\delta t$ as $\bar{t}$ is increased. As noted in Ref. [10], all the quantities used here are readily obtained in experiments, and the method of data analysis should be well suited for experimental analysis as well.

The asymptotic scaling predicted for large and small $\bar{t}$ can also be tested. First, the characteristic time difference $\delta t_{c}$ defined by $\operatorname{Cov}\left(\delta t_{c}, \bar{t}\right)=\frac{1}{2} \operatorname{Cov}(0, \bar{t})$ can be found for fixed ranges of $\bar{t}$. The measured $\delta t_{c}$, found by linear interpolation, is presented as a function of $\bar{t}$ on a log-log scale in Fig. 8. The results show obvious power-law behavior at both small and large $\bar{t}$. A least-squares fit for $\bar{t}<50$ gives an exponent of $1.02 \pm 0.02$, which agrees with our expectation that in this regime the two-time structure factor scales with $\delta t / \bar{t}$ (Sec. II B 1). Least-squares fitting for $\bar{t}>300$ gives an exponent of $0.66 \pm 0.01$, in excellent agreement with the theoretical prediction of $1-n=2 / 3$ expected from $z=\delta t / \bar{t}^{1-n}$ (Sec. II B 2). The fit also indicates that the characteristic $\bar{t}$

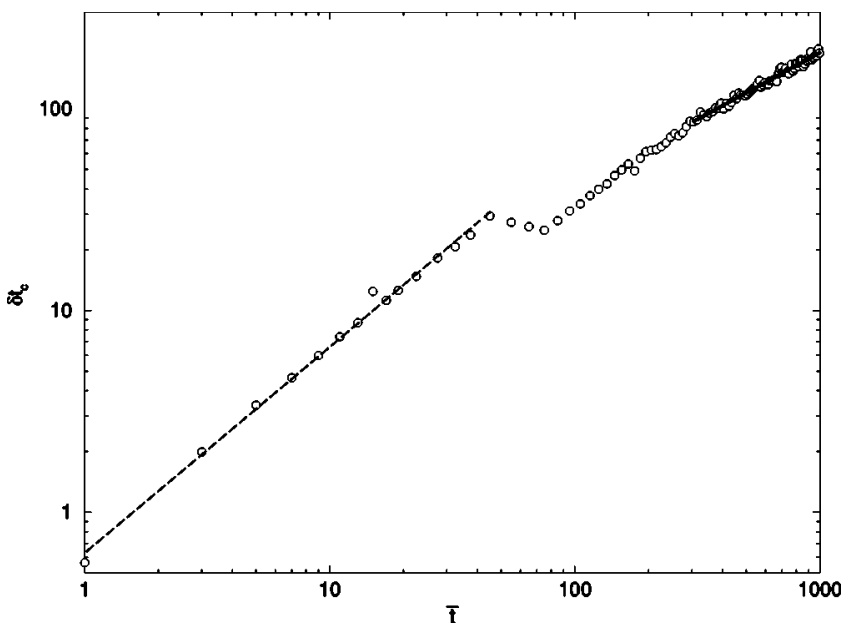

FIG. 8. Characteristic time difference $\delta t_{c}$ defined by $\operatorname{Cov}\left(\delta t_{c}, \bar{t}\right)=(1 / 2) \operatorname{Cov}(0, \bar{t})$. The lines are least-squares fits to power-law behavior. For $\bar{t}<50$ (dashed line) the fit gives an exponent $1.02 \pm 0.02$, which agrees with our expectation for small $\bar{t}$. For $\bar{t} \geqslant 300$ (solid line) the least-squares fit gives an exponent 0.66 \pm 0.01 . This agrees with what is expected from the large- $\bar{t}$ scaling variable $z=\delta t / \bar{t}^{1-n}$ with $1-n=2 / 3$, as well as with a recent XIFS experiment [8].

for crossover between these regimes is $\bar{t}_{c} \sim 60$, which corresponds to the shoulder in the structure factor (see Figs. 6 and $10)$.

The scaling function $\Phi_{d}(z)$ associated with the speckle covariance at large $\bar{t}$ is shown in Fig. 9(a) for several $\bar{t}$ $>700$. Here the normalization was determined from the asymptotic data, where it appears that $\left(2 \pi C_{1}\right)^{2} \approx 32$. The collapse of the data is quite good, and the agreement with the theoretical scaling form $\Phi_{2}(z)$ from Eq. (28) is also excellent for $z \lesssim 4.5$. These results are shown on a linear-log scale in Fig. 9(b), along with the scaled two-time structure factor data for the same $\bar{t}$ (drawn as filled symbols). The fitting parameter $n C_{2} \approx 0.62$ was determined from a nonlinear fit to the two-time structure factor data. Fitting to the covariance data did not significantly increase the agreement with $\Phi_{2}(z)$. This theoretical scaling form gives much better overall agreement with the data than more conventional choices, such as a Gaussian or Lorentzian. Beyond $z \approx 4$ the intensity covariance is quite small. However, as $z$ increases further it becomes larger than the squared structure factor, which remains reasonably well described by $\Phi_{2}(z)$ for all $z$ studied. This deviation signals the breakdown of the Gaussian decoupling approximation, and is the same deviation seen at large $t_{1}$ in Fig. 6.

Measurements that include intensities only for two measurement times, $\tau_{1}$ and $\tau_{2}$, correspond to rays of slope $\tau_{1} / \tau_{2}$ in the $\left(t_{1}, t_{2}\right)$ plane. The wave vector then serves as the parameter indicating position along the ray, and the values of the intensity covariance along the $t_{1}=t_{2}$ ray are the structure factor squared. Figure 10(a) shows the simulation estimates for the scaled structure factor $F_{1}(t)$ for several different times on a $\log -\log$ scale. The collapse for different $\tau$ is quite reasonable. The two straight lines are provided as a reference to the expected asymptotic behavior. The dashed line in Fig. 10 (a) indicates the slope corresponding to the power law $S$ 

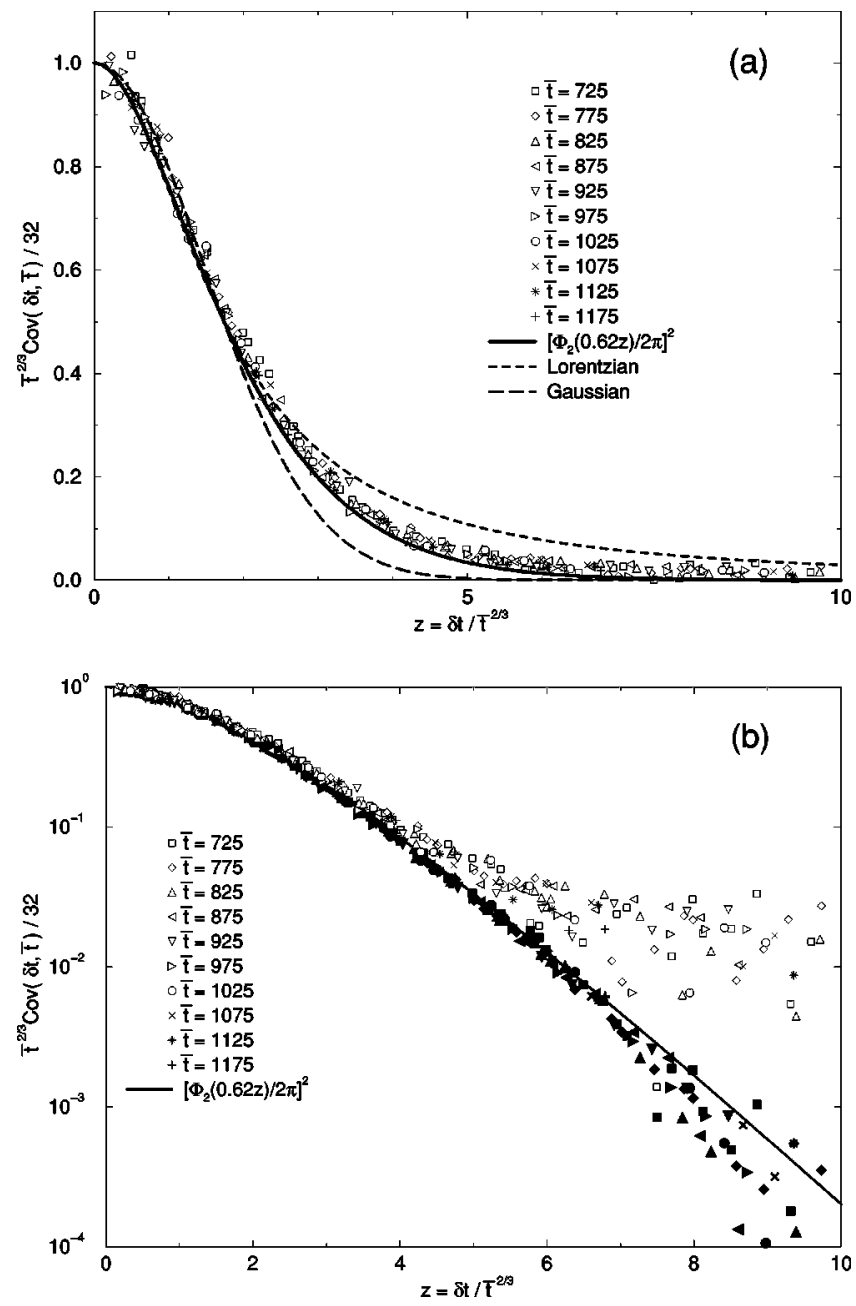

FIG. 9. Scaling of the covariance vs $\delta t / \bar{t}^{1-n}$ for several values of $\bar{t}$ is shown. (a) Linear scale. (b) Linear-log scale, where data for the two-time structure factor are also shown (filled symbols). The solid curve is the theoretical form, Eq. (27). The long-dashed and short-dashed curves in (a) are Gaussian and Lorentzian forms fitted to the same half width. Inspection of data indicates $\left(2 \pi C_{1}\right)^{2} \approx 32$, and numerical fitting to the two-time structure factor data gives $n C_{2} \approx 0.62$. The data collapse is good, confirming that there is a single master curve for the speckle covariance in the large- $\bar{t}$ limit. The deviation of the covariance data from the two-time structure factor for $z \gtrless 4.5$ indicates the gradual breakdown of the Gaussian decoupling approximation for these simulations.

$\propto k^{\beta(n)}$ or $F_{1} \propto t^{n(\beta(n)+d)}$, with $\beta=4$ for model $B$ as discussed in Sec. II B 1. The solid line corresponds to the Porod's law result $S \propto k^{-(d+1)}$ (or $F_{1} \propto t^{-n}$ ) at high wave vectors, which is an important part of the moving-interface model associated with the asymptotic scaling form $\Phi_{d}(z)$. The simulated structure factor reproduces both behaviors. The inset is the representation of the master curve $F_{1}^{\prime}$ in terms of the scaled wave vector $q=k[B \tau]^{1 / 3}$. The deviation from the expected power law at small $q$ has been observed in other simulations and is expected to vanish as $\tau \rightarrow \infty$ [15].

In the case of a nonconserved order parameter, the analytic theory of Ohta, Jasnow, and Kawasaki (OJK) [14,35] gives excellent quantitative agreement for the one-time structure factor $[36,37]$. The YJ extension of that theory $[16]$ to two-time correlations gives good agreement for the intensity
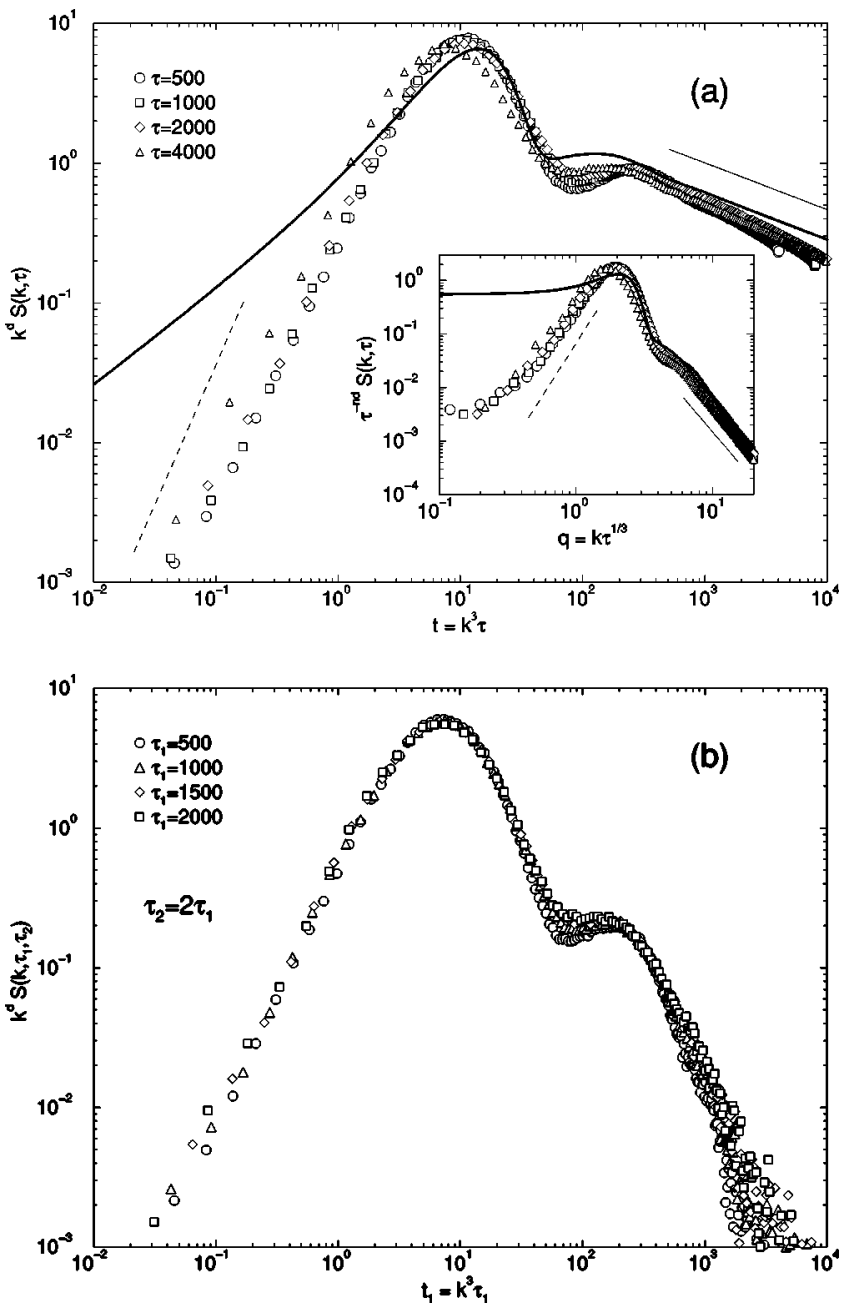

FIG. 10. Scaling collapse of the structure factor. (a) Scaling of the one-time structure factor for several different times presented on a $\log -\log$ scale vs $t=k^{3} \tau$. The slopes of the straight lines correspond to the expected power laws, $S(k, \tau) \propto k^{4}$ at small wave vectors (dashed line) and $\propto k^{-(d+1)}$ at large wave vectors (solid line), respectively. The heavy solid curve is the theory of Yeung, Oono, and Shinozaki [39]. The inset shows the equivalent scaling collapse of Eq. (3). (b) Scaling of the two-time structure factor for fixed $\tau_{1} / \tau_{2}=1 / 2$ and several different $\tau_{1}$. This corresponds to a cut through $\operatorname{Cov}\left(k, \tau_{1}, \tau_{2}\right)$ along a line of slope $\frac{1}{2}$. The $\tau_{1}=2000$ data represented by squares are the same as those represented by squares in Fig. 6(b).

covariance except at very large $\delta t[10]$. No comparably successful theory exists for the conserved order-parameter case considered here. Approximate forms for the one-time structure factor (none of which has the expected $k^{4}$ behavior at small $k$ ) have been proposed by Ohta and Nozaki [38] and by Tomita [39], Yeung, Oono, and Shinozaki [40]. Of these, we find the best agreement for the latter result $[39,40]$. The heavy solid curve in Fig. 10(a) represents this approximate structure factor as obtained from the differential-equation formulation in $k$ space found in Ref. [40]. This theory extends a model of interface motion to phase separation and recovers $n=1 / 3$. Here it has been fit numerically to the simulation data using one adjustable parameter. While it gives reasonable agreement at small distances, it fails at larger distances as seen by the deviations at small $t$ in Fig. 10(a). 

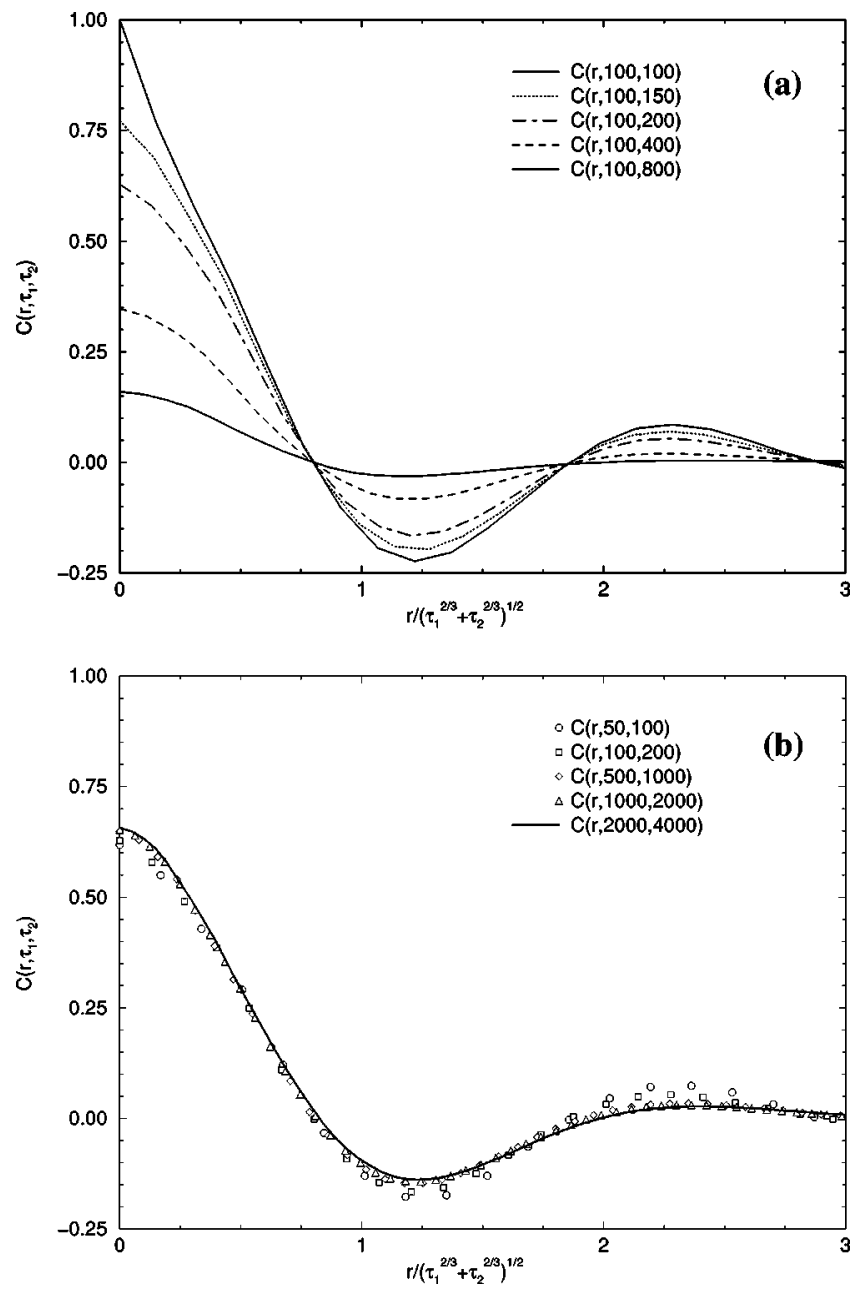

FIG. 11. Scaling of the two-time correlation function. (a) For $\tau_{1}=100$ and various $\tau_{2}$. The zero crossing points remain approximately stationary while the amplitude of oscillations decreases monotonically. (b) For several $\tau_{1} / \tau_{2}=\frac{1}{2}$. A scaling form appears to be approached at later times. The solid curve, the simulation result involving the latest time, is taken as an estimate of the scaling function for this ratio of times.

There is also noticeable disagreement around the shoulder at high $t$. A better analytic model for the locally conserved dynamics of phase separation is clearly still needed.

The scaling of the two-time structure factor along the ray $\tau_{1} / \tau_{2}=1 / 2$ is shown in Fig. 10(b) for several $\tau_{1}$. The data collapse is again quite good. The largest change from the one-time structure factor shown in Fig. 10(a) is the disappearance of the Porod tail at large wave vector, which is consistent with the idea that moving interfaces are controlling the two-time properties of the system.

It is also interesting to consider scaling for the two-time order-parameter correlation function for large $r$, where it is not linear in $r$ and the scattering is not governed by Porod's law. In Sec. II B 1, we argued that the scaling variables should be $r /[B \bar{\tau}]^{n}$ and $\delta \tau / \bar{\tau}$, and this gives quite good agreement with the simulations. However, an equivalent set of scaled variables gives what we believe is a more natural form for the correlation function. It is inspired by the YJ correlation function appropriate to model $A[10,16]$, Eq. (A1), where scaled lengths enter as $r^{2} /\left[R^{2}\left(\tau_{1}\right)+R^{2}\left(\tau_{2}\right)\right]$.

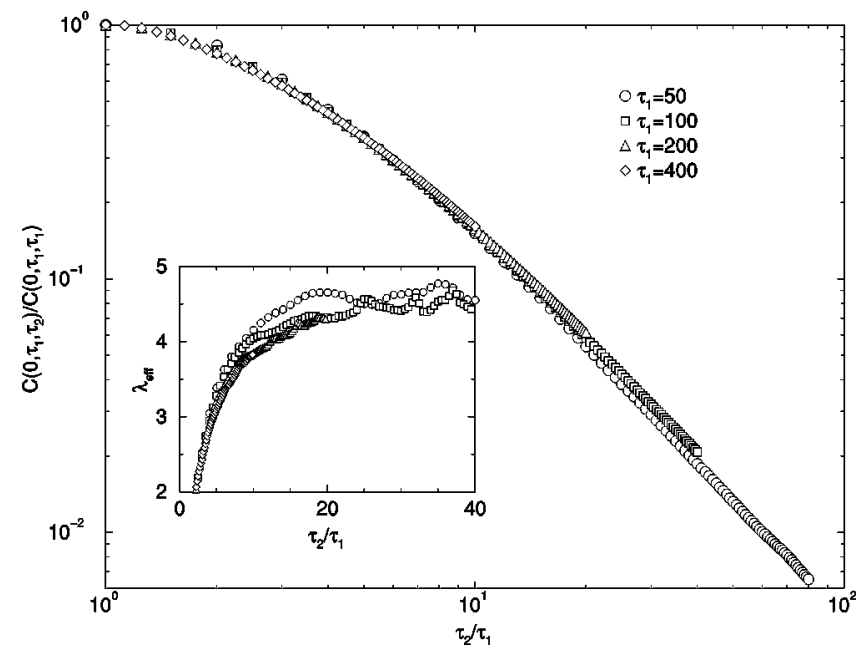

FIG. 12. Log-log plot of the autocorrelation vs $\tau_{2} / \tau_{1}$ for several values of $\tau_{1}$. Least-squares fitting for $\tau_{1}=50, \tau_{2}>2000$ gives a slope of $1.49 \pm 0.01$, which corresponds to $\lambda \approx 4.5$. This value violates the upper bound $\lambda \leqslant d$ conjectured by Fisher and Huse [40], but is consistent with the constraint $\lambda \geqslant(d / 2)+2$ derived by Yeung, et al. [41] for conserved systems.

(This is a consequence of the assumption of a Gaussian auxiliary field.) The simulated two-time order-parameter correlation function for model $B$ is well scaled out to quite large $r$ using

$$
C\left(\mathbf{r}, \tau_{1}, \tau_{2}\right)=\widetilde{C}\left(\frac{r}{B^{n} \sqrt{\tau_{1}^{2 n}+\tau_{2}^{2 n}}}, \frac{\tau_{1}}{\tau_{2}}\right) .
$$

The results for fixed $\tau_{1}$ and several $\tau_{2}$ are presented in Fig. 11(a). The zeros of the correlation function are approximately stationary with respect to $x=r / \sqrt{\tau_{1}^{2 / 3}+\tau_{2}^{2 / 3}}$ for the range of times shown here. The scaling becomes less good for much smaller values of $\tau_{1} / \tau_{2}$. As the ratio $\tau_{1} / \tau_{2}$ decreases, the amplitude of the oscillations also decreases. The collapse of $C\left(r, \tau_{1}, \tau_{2}\right)$ onto $\widetilde{C}\left(x, \tau_{1} / \tau_{2}\right)$ for several pairs of $\tau_{1}$ and $\tau_{2}$ is shown in Fig. 11(b). The simulation results appear to converge for later times, and the collapse is quite good for $\tau_{1} \geqslant 500$. The solid curve represents the simulation estimate of the master curve for this ratio of times with $\tau_{2}$ $=4000$.

One last property of phase-separating materials to consider is the autocorrelation of the order parameter, $C\left(0, \tau_{1}, \tau_{2}\right)$, for $\tau_{2} \gg \tau_{1}$. In this regime (the opposite of the small time-difference regime emphasized elsewhere in this paper), Fisher and Huse [41] have argued that the autocorrelation should be described by a power-law exponent $\lambda$ as

$$
C\left(0, \tau_{1}, \tau_{2}\right) \propto\left(\frac{R\left(\tau_{1}\right)}{R\left(\tau_{2}\right)}\right)^{\lambda} .
$$

The YJ correlation function for model $A$ (see the Appendix) predicts $\lambda=d / 2$. This is a weakness of the YJ theory; the value observed in experiments and simulations on model $A$ indicate $\lambda$ is clearly larger [10]. For model $B$, Yeung, Rao, and Desai (YRD) [42] found $\lambda \approx 4$ for $\tau_{1}$ in the scaling regime from a numerical simulation similar to the one presented here. The autocorrelation for the present simulations 
is shown in Fig. 12, where the results have been normalized by $C\left(0, \tau_{1}, \tau_{1}\right)$ to show the collapse for different $\tau_{1}$. The inset shows the values of $\lambda$ estimated from the derivative of $C\left(0, \tau_{1}, \tau_{2}\right)$ as a function of $\tau_{2} / \tau_{1}$. For the two earliest $\tau_{1}, \lambda$ appears to have converged to its asymptotic value. A leastsquares fit to the latest times $\left(\tau_{2}>2000\right)$ for $\tau_{1}=50$ gives $\lambda=4.47 \pm 0.03$. Both our result and that of YRD violate the upper bound $\lambda \leqslant d$ conjectured by Fisher and Huse for phaseordering systems [41]. They are consistent with the lower bound $\lambda \geqslant(d / 2)+2$ found for spinodal decomposition in $d$ $\geqslant 2$ by YRD [42]. Lee and Rutenberg [43] found the same bound for small, but nonvanishing, minority-phase volumes. They point out that correlations in the minority domains accelerate the decay of the autocorrelation.

\section{CONCLUSIONS}

The scattering intensity for two-dimensional systems undergoing spinodal decomposition has been investigated using numerical integration of the Cahn-Hilliard-Cook equation. The results indicate that the two-time structure factor can be measured experimentally via the speckle-intensity covariance at $\mathbf{k} \neq \mathbf{0}$. The connection between the two quantities is established directly by comparing numerical estimates of both and indirectly by verifying the exponential probability distribution of the scattering intensity. For the present simulations, the equality between the two-time structure factor and the speckle-intensity covariance is observed to break down at $z \gtrsim 4.5$. These properties were also observed in previous simulations for nonconserved dynamics [10], and they should be common to any heterogeneous material with a large number of independent domains.

An important conclusion of our study is that the scaling behavior of the speckle-intensity covariance at both large and small $\bar{t}$ can be obtained from geometric arguments based on a simple moving-interface picture. These scaling results depend on the dynamics only implicitly through the values of the dynamic exponent $n$ and the exponent $\beta(n)$, which gives the asymptotic small- $k$ behavior of the one-time structure factor. Specifically, we have argued that the asymptotic scaling of the two-time structure factor for large $\bar{t}$ is in terms of $z=\delta t / \bar{t}^{1-n}$, with $1-n=2 / 3$ for spinodal decomposition. For small $\bar{t}$, on the other hand, the appropriate scaling variable is $\delta t / \bar{t}$. These behaviors were recently confirmed by XIFS studies Ref. [8,44] of spinodal decomposition and demonstrate that experimentally observable aspects of both the short and long length- and time-scale regimes of phase separation can be described by relatively simple models of interface motion. A simple form motivated by the YeungJasnow theory of phase ordering gives good agreement with simulation results for the correlation function.

Even though much of the large $\mathbf{k}$ behavior of model $B$ can be understood theoretically, a complete theory of phase separation, even one that just describes the one-time structure factor, is still needed.

\section{ACKNOWLEDGMENTS}

We would like to thank G. Korniss, G. B. Stephenson, F. Bley, F. Livet, A. Rutenberg, and T. Kawakatsu for useful discussions. Research at Florida State University was supported by the Center for Materials Research and Technology, by the Supercomputer Computations Research Institute (under U.S. Department of Energy Contract No. DE-FC0585ER25000), and by the National Science Foundation under Grant No. DMR-9634873. Research at McGill University was supported by the Natural Sciences and Engineering Research Council of Canada and le Fonds pour la Formation de Chercheurs et l'Aide á la Recherche du Québec. Supercomputer time at the U.S. National Energy Research Scientific Computing Center was made available by the U.S. Department of Energy.

\section{APPENDIX}

In fact, $G(x)=\sqrt{1+x^{2}}$ is the form which results from the YJ theory for model $A[10,16]$. Those authors introduced the two-time order-parameter correlation function [16]

$$
\begin{aligned}
C_{\mathrm{YJ}}\left(r, \tau_{1}, \tau_{2}\right)= & \frac{2}{\pi} \arcsin \left[\left(\frac{2 R\left(\tau_{1}\right) R\left(\tau_{2}\right)}{R\left(\tau_{1}\right)^{2}+R\left(\tau_{2}\right)^{2}}\right)^{d / 2}\right. \\
& \left.\times \exp \left(\frac{-r^{2}}{R\left(\tau_{1}\right)^{2}+R\left(\tau_{2}\right)^{2}}\right)\right] .
\end{aligned}
$$

In this case, $R(\tau)=[B \tau]^{n}$ with $n=1 / 2$ and $B=4(d-1) / d$. Expanding $R\left(\tau_{1}\right)$ and $R\left(\tau_{2}\right)$ in terms of $\delta \tau$ and $\bar{\tau}$ and expressing the argument of the arcsin to lowest order in $\delta \tau / \bar{\tau}$ and $r /[B \bar{\tau}]^{n}$, we get

$$
C_{\mathrm{YJ}}(r, \delta \tau, \bar{\tau})=\frac{2}{\pi} \arcsin \left[1-\frac{d}{16}\left(\frac{\delta \tau}{\bar{\tau}}\right)^{2}-\frac{1}{2}\left(\frac{r}{[B \bar{\tau}]^{n}}\right)+\cdots\right] .
$$

The expansion for arguments near unity, $\arcsin (x) \approx \pi / 2$ $-\sqrt{2(1-x)}$, then gives

$$
C_{\mathrm{YJ}}(r, \delta \tau, \bar{\tau}) \approx 1-\sqrt{\frac{d}{8}} \frac{2}{\pi} \frac{n \zeta}{\bar{\tau}^{1 / 2}} \sqrt{1+\left(\sqrt{\frac{d}{8}} \frac{r}{B^{1 / 2} n \zeta}\right)^{2}} .
$$

This correlation function corresponds to Eq. (21) with $C_{1}$ $=2 / \pi, C_{2}=\sqrt{d / 8}$, and $G(x)=\sqrt{1+x^{2}}$. In Ref. [10], the resulting analytic scaling form for the two-time structure factor at large $\bar{t}, \Phi_{d}(z)$ given by Eq. (27), was obtained for model $A$ by a different method utilizing the full form, Eq.(A1). That approach also yielded a small- $\bar{t}$ result corresponding to the Taylor expansion Eq. (15) and the corresponding condition Eq. (17).
[1] J. Feder, Fractals (Plenum Press, New York, 1988).

[2] B. Chu, Laser Light Scattering (Academic Press, New York, 1974).

[3] L. Mandel and E. Wolf, Optical Coherence and Quantum Op- tics (Cambridge University Press, Cambridge, 1995).

[4] S. B. Dierker, R. Pindak, R. M. Fleming, I. K. Robinson, and L. Berman, Phys. Rev. Lett. 75, 449 (1995).

[5] B. Chu, Q.-C. Ying, F.-J. Yeh, A. Patkowski, W. Steffen, and 
E. W. Fischer, Langmuir 11, 1419 (1995).

[6] S. Brauer, G. B. Stephenson, M. Sutton, R. Brüning, E. Dufresne, S. G. J. Mochrie, G. Grübel, J. Als-Nielsen, and D. L. Abernathy, Phys. Rev. Lett. 74, 2010 (1995).

[7] S. G. J. Mochrie, A. M. Mayes, A. R. Sandy, M. Sutton, S. Brauer, G. B. Stephenson, D. L. Abernathy, and G. Grübel, Phys. Rev. Lett. 78, 1275 (1997).

[8] A. Malik, A. R. Sandy, L. B. Lurio, G. B. Stephenson, S. G. J. Mochrie, I. McNulty, and M. Sutton, Phys. Rev. Lett. 81, 5832 (1998).

[9] G. Brown, P. A. Rikvold, and M. Grant, Physica A 239, 363 (1997).

[10] G. Brown, P. A. Rikvold, M. Sutton, and M. Grant, Phys. Rev. E 56, 6601 (1997).

[11] A comprehensive review is given by J. D. Gunton, M. San Miguel, and P. S. Sahni, in Phase Transitions and Critical Phenomena, edited by C. Domb and J. L. Lebowitz (Academic, London, 1983), Vol. 8. Ideas of scaling in domain growth follow similar ideas in critical dynamics, P. C. Hohenberg and B. I. Halperin, Rev. Mod. Phys. 49, 435 (1977). A recent specialized review is given by A. J. Bray, Adv. Phys. 43, 357 (1994)

[12] M. Tomozawa, in Encyclopedia of Materials Science and Engineering, edited by M. B. Bever (Pergamon Press, Oxford, 1986), p. 3493.

[13] A. Stuart and J. K. Ord, Kendall's Advanced Theory of Statistics, 5th ed. (Griffin, London, 1987), Vol. 1.

[14] T. Ohta, D. Jasnow, and K. Kawasaki, Phys. Rev. Lett. 49, 1223 (1982).

[15] C. Yeung, Phys. Rev. Lett. 61, 1135 (1988).

[16] C. Yeung and D. Jasnow, Phys. Rev. B 42, 10523 (1990).

[17] G. Porod, in Small Angle X-ray Scattering, edited by O. Glatter and L. Kratky (Academic, New York, 1983); A. Guinier and G. Fournet, Small-Angle Scattering of X-rays (Wiley, New York, 1955).

[18] P. Debye, H. R. Anderson, and H. Brumberger, J. Appl. Phys. 28, 679 (1957).

[19] H. Tomita, Prog. Theor. Phys. 72, 656 (1984); 75, 482 (1986).

[20] H. Tomita, in Formation Dynamics and Statistics of Patterns, edited by K. Kawasaki, M. Suzuki, and A. Onuki (World Scientific, Singapore, 1990).

[21] A. Erdélyi, Asymptotic Expansions (Dover, New York, 1956).
[22] P. A. Rikvold and G. Brown (unpublished).

[23] G. Korniss (private communication).

[24] R. Wong, Asymptotic Approximations of Integrals (Academic, Boston, 1989), Chap. IV.

[25] I. S. Gradshteyn and I. M. Ryzhik, Tables of Integrals, Series, and Products (Academic, New York, 1980), p. 721 [6.645.2].

[26] Handbook of Mathematical Functions, edited by M. Abramowitz and I. A. Stegun, Natl. Bur. Stand. (U.S. GPO, Washington, DC, 1970), p. 444.

[27] J. W. Cahn and H. E. Hilliard, J. Chem. Phys. 28, 258 (1958).

[28] H. E. Cook, Acta Metall. 18, 297 (1970).

[29] M. Grant, M. San Miguel, J. Viñals, and J. D. Gunton, Phys. Rev. B 31, 3027 (1985).

[30] Explicitly: $\psi \rightarrow \psi \sqrt{a / u}, r \rightarrow r \sqrt{c / a}$, and $\tau \rightarrow \tau c /\left(2 M a^{2}\right)$.

[31] Y. Oono and S. Puri, Phys. Rev. Lett. 58, 836 (1987).

[32] H. Tomita, Prog. Theor. Phys. 85, 47 (1991).

[33] The maximum dimensionless wave number allowed by the discretization of the continuum equations is $\pi$.

[34] A. Shinozaki and Y. Oono, Phys. Rev. E 48, 2622 (1993).

[35] T. Ohta, Ann. Phys. (N.Y.) 158, 31 (1984).

[36] G. Brown, P. A. Rikvold, and M. Grant, Phys. Rev. E 58, 5501 (1998).

[37] The result for the amplitude in Porod's law [18] was regrettably rendered incorrectly after Eq. (9) in Ref. [36]. Using the notation of that paper, the result should be $F(q)$ $=2^{d} \pi^{(d-1) / 2} \Gamma[(d+1) / 2] \alpha q^{-(d+1)}$, consistent with the expressions in Eq. (24) of the present paper and Eq. (5.9) of Ref. [10].

[38] T. Ohta and H. Nozaki, in Space-Time Organization in Macromolecular Fluids, Vol. 51 of Springer Series in Chemical Physics, edited by F. Tanaka, M. Doi, and T. Ohta (Springer, Berlin, 1989).

[39] H. Tomita, Prog. Theor. Phys. 90, 521 (1993).

[40] C. Yeung, Y. Oono, and A. Shinozaki, Phys. Rev. E 49, 2693 (1994).

[41] D. S. Fisher and D. A. Huse, Phys. Rev. B 38, 373 (1988).

[42] C. Yeung, M. Rao, and R. Desai, Phys. Rev. E 53, 3073 (1996).

[43] B. P. Lee and A. D. Rutenberg, Phys. Rev. Lett. 79, 4842 (1997).

[44] F. Livet, F. Bley, R. Caudron, D. Abernathy, C. Detlefs, G. Grübel, and M. Sutton (unpublished). 